\title{
Low-algorithmic-complexity entropy-deceiving graphs
}

\author{
Hector Zenil* \\ Information Dynamics Lab, Unit of Computational Medicine, Department of Medicine Solna, Center for Molecular Medicine, SciLifeLab, \\ Karolinska Institute, Stockholm 171 76, Sweden; \\ Department of Computer Science, University of Oxford, Oxford OX1 3QD, United Kingdom; \\ and Algorithmic Nature Group, LABoRES, Paris 75006, France
}

Narsis A. Kiani

Information Dynamics Lab, Unit of Computational Medicine, Department of Medicine Solna, Center for Molecular Medicine, SciLifeLab, Karolinska Institute, Stockholm 171 76, Sweden and Algorithmic Nature Group, LABoRES, Paris 75006, France

Jesper Tegnér

Biological and Environmental Sciences and Engineering Division, Computer, Electrical and Mathematical Sciences and Engineering Division, King Abdullah University of Science and Technology (KAUST), Thuwal 23955 - 6900, Kingdom of Saudi Arabia and Unit of Computational Medicine, Department of Medicine Solna, Center for Molecular Medicine, SciLifeLab, Karolinska Institute, Stockholm 171 76, Sweden

(Received 14 October 2016; revised manuscript received 19 March 2017; published 7 July 2017)

\begin{abstract}
In estimating the complexity of objects, in particular, of graphs, it is common practice to rely on graphand information-theoretic measures. Here, using integer sequences with properties such as Borel normality, we explain how these measures are not independent of the way in which an object, such as a graph, can be described or observed. From observations that can reconstruct the same graph and are therefore essentially translations of the same description, we see that when applying a computable measure such as the Shannon entropy, not only is it necessary to preselect a feature of interest where there is one, and to make an arbitrary selection where there is not, but also more general properties, such as the causal likelihood of a graph as a measure (opposed to randomness), can be largely misrepresented by computable measures such as the entropy and entropy rate. We introduce recursive and nonrecursive (uncomputable) graphs and graph constructions based on these integer sequences, whose different lossless descriptions have disparate entropy values, thereby enabling the study and exploration of a measure's range of applications and demonstrating the weaknesses of computable measures of complexity.
\end{abstract}

DOI: 10.1103/PhysRevE.96.012308

\section{THE USE OF SHANNON ENTROPY IN NETWORK PROFILING}

One of the major challenges in modern physics is to provide proper and suitable representations of network systems for use in fields ranging from physics [1] to chemistry [2]. A common problem is the description of order parameters with which to characterize the "complexity of a network." Graph complexity has traditionally been characterized using graphtheoretic measures such as the degree distribution, clustering coefficient, edge density, and community or modular structure.

More recently, networks have also been characterized using classical information theory. One problem in this area is the interdependence of many graph-theoretic properties, which makes measures more sophisticated than single-property measurements [3] difficult to come by. The standard way to

\footnotetext{
*hector.zenil@algorithmicnaturelab.org; http://algorithmicnature.org/
}

Published by the American Physical Society under the terms of the Creative Commons Attribution 4.0 International license. Further distribution of this work must maintain attribution to the author(s) and the published article's title, journal citation, and DOI. address this is to generate graphs that have a certain specific property while being random in all other aspects, in order to check whether or not the property in question is typical among an ensemble of graphs with otherwise seemingly different properties.

Approaches using measures based upon the Shannon entropy's claim to quantify the information content of a network [4] as an indication of its "typicality" are based on the assumption of associated ensembles provided by the entropy evaluation: the more random, the more typical. The claim is that one can construct a "null model" that captures some aspects of a network (e.g., graphs that have the same degree distribution) and see how different the network is from the null model as regards particular features, such as the clustering coefficient, graph distance, and other features of interest. The procedure aims at producing an intuition of an ensemble of graphs that are assumed to have been sampled uniformly at random from the set of all graphs with the same property to determine whether such a property occurs with a high or a low probability. If the graph is not significantly different, statistically, from the null model, then the graph is claimed to be as "simple" as the null model; otherwise, the measure is claimed to be a lower bound on the "complexity" of the graph as an indication of its random versus causal nature. 
Here we highlight some serious limitations of these approaches that are often neglected and provide pointers to approaches that are complementary to Shannon entropy, in order to partially circumvent some of the aforesaid limitations by combining it with a measure of local algorithmic complexity that better captures the recursive and thus causal properties of an object-in particular, a network-beyond statistical properties.

One of the most popular applications of the entropy is to graph the degree distribution, as first suggested and introduced in [5]. Similar approaches have been adopted in areas such as chemical graph theory and computational systems biology [6] as functions of the layered graph degree distribution under certain layered coarse-graining operations (sphere covers), leading to the hierarchical application of the entropy, a version of the graph traversal entropy rate. In chemistry, for example, the Shannon entropy of a graph degree sequence has been used as a profiling tool to characterize molecular complexity [7-11].

While the application of the entropy to graph degree distributions has been relatively more common, the same entropy has also been applied to other graph features, such as functions of their adjacency matrices [12], and to distance and Laplacian matrices [13]. Even more recently, the Shannon entropy on adjacency matrices was used to attempt the discovery of CRISPR regions in an interesting transformation of DNA sequences into graphs [14]. A survey contrasting adjacency-matrix-based (walk) entropies and other entropies (e.g., on degree sequence) is offered in [12]. It finds that adjacency-based entropies are more robust vis-à-vis graph size and are correlated with the graph algebraic properties, as these are also based on the adjacency matrix (e.g., graph spectrum).

Finally, hybrid measures have been used, such as the graph heterogeneity index [15] as a function of degree sequence and the Laplacian matrix, where some of the limitations of quantifying only the diversity of the degree distribution, i.e., its entropy (or that of any graph measure as a function of the entropy of the degree distribution), have been identified.

It is thus of the greatest interest to researchers in physics, chemistry, and biology to understand the reach, limits, and interplay of measures of entropy, in particular, as applied to networks. Likewise, it is of great interest to understand how unserviceable for extracting causal content-as opposed to randomness - the use of entropy as a measure of randomness, complexity, or information content can be. The use of entropy has, however, been extended, because its numerical calculation is computationally very cheap compared to richer, but more difficult to approximate universal measures of complexity, which are better qualified to capture more general properties of graphs. Some of these properties to be captured are related to the nature of the graph-generating mechanisms, which were what most of the previously utilized measures were supposed to quantify in the first place, in one way or another, from the introduction of the first random graph model by Erdös and Rényi [16] to the most popular models such as "scale-freeness" [17] and more recent ones such as network randomness typicality [4].

\section{NOTATION AND BASIC DEFINITIONS}

Definition II.1. A graph is an ordered pair $G=(V, E)$ comprising a set $V$ of nodes or vertices and a set $E$ of edges or links, which are two-element subsets of $V$.

Definition II.2. A graph $G$ is labeled when the vertices are distinguished by labels $u_{1}, u_{2}, \ldots, u_{n}$, with $n=|V(G)|$ the cardinality of the set $V(G)$.

Definition II.3. Graphs $G$ and $H$ are isomorphic if there is a bijection between the vertex sets of $G$ and $H, \lambda: V(G) \rightarrow$ $V(H)$, such that any two vertices $u$ and $v \in V(G)$ are adjacent in $G$ if and only if $\lambda(u)$ and $\lambda(v)$ are adjacent in $H$.

Definition II.4. The degree of a node $v$, denoted $d(v)$, is the number of (both incoming and outgoing) links to other nodes, and $d$ is the unordered list of all $v \in V(G)$.

Definition II.5. An Erdös-Rényi (E-R) graph $G(n, p)$ is a graph of size $n$ constructed by connecting nodes randomly with probability $p$ independent of every other edge.

Usually E-R graphs are assumed to be nonrecursive (i.e., truly random), but E-R graphs can be constructed recursively using pseudorandom generating algorithms.

\section{A. Graph entropy}

One of the main objectives behind the application of the Shannon entropy is the characterization of the randomness or "information content" of an object such as a graph. Here we introduce graphs with interesting deceptive properties, particularly, disparate entropy (rate) values for the same object looked at from different perspectives, revealing the inadequacy of classical information-theoretic approaches to graph complexity (Figs. 1-3).

Central to information theory is the concept of Shannon's information entropy, which quantifies the average number of bits needed to store or communicate the statistical description of an object. For an ensemble $X\left(R, p\left(x_{i}\right)\right)$, where $R$ is the set of possible outcomes (the random variable), $n=|R|$ and $p\left(x_{i}\right)$ is the probability of an outcome in $R$. The Shannon entropy of $X$ is then given by

Definition II.6.

$$
H(X)=-\sum_{i=1}^{n} p\left(x_{i}\right) \log _{2} p\left(x_{i}\right),
$$

which implies that to calculate $H(X)$ one has to know or assume the mass distribution probability of ensemble $X$. One caveat regarding Shannon's entropy is that one is forced to make an arbitrary choice regarding granularity. Take, for example, the bit string 01010101010101. The Shannon entropy of the string at the level of single bits is maximal, as there are the same number of $1 \mathrm{~s}$ and $0 \mathrm{~s}$, but the string is clearly regular when two-bit (nonoverlapping) blocks are taken as basic units, in which case the string has minimal complexity because it contains only one symbol (01) among four possibilities $(00,01$, $10,11)$. A generalization consists in taking into consideration all possible "granularities" or the entropy rate:

Definition II.7. Let $\operatorname{Pr}\left(s_{i}, s_{i+1}, \ldots, s_{i+L}\right)=\operatorname{Pr}(s)$ with $|s|=$ $L$ denote the joint probability over blocks of $L$ consecutive symbols. Let the Shannon entropy rate [21] (also known as the granular entropy or $n$-gram entropy) of a block of $L$ 

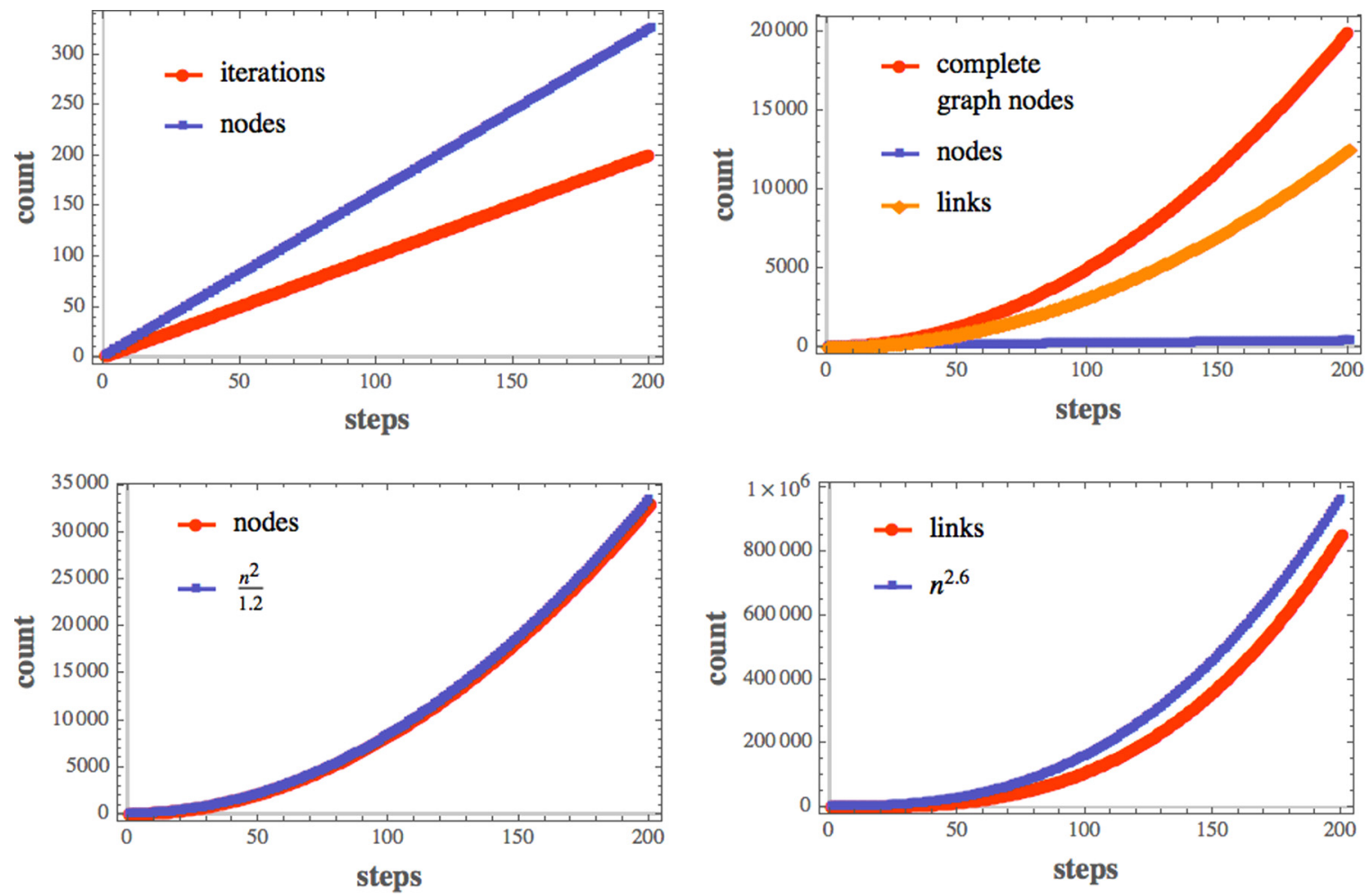

FIG. 1. Basic node and link growth properties and corresponding fitted (polynomial) lines. The relation between node and link growth determines the edge density, which at the limit is 0 .

consecutive symbols—denoted $H(L)$-be

$$
H_{L}(s)=-\sum_{s 1 \in A} \ldots \sum_{s_{L} \in A} \operatorname{Pr}\left(s_{1}, \ldots, s_{L}\right) \log _{2} \operatorname{Pr}\left(s_{1}, \ldots, s_{L}\right) .
$$

Thus to determine the entropy rate of the sequence, we estimate the limit when $L \rightarrow \infty$. It is not hard to see, however, that $H_{L}(s)$ will diverge as $L$ tends to $\infty$ if the number of symbols increases, but if applied to a binary string $H_{L}(s)$, it will reach a minimum for the granularity in which a statistical regularity is revealed.

The Shannon entropy [21] of an object $s$ is simply $H_{L}(s)$ for fixed block size $L=i$, so we can drop the subscript. We can define the Shannon entropy of a graph $G$, with respect to $i$, by:

Definition II.8.

$$
H(G, P)=-\sum_{i}^{|G|} P\left(G_{i}\right) \log _{2} P\left(G_{i}\right)
$$

where $P$ is a probability distribution of $G_{i}, i$ is a feature of interest of $G$, e.g., the edge density, degree sequence, number of overrepresented subgraphs/graphlets (graph motifs), and so on. When $P$ is the uniform distribution (every graph of the same size is equally likely), it is usually omitted as a parameter of $H$.

The most common applications of the entropy to graphs are to the degree sequence distribution and edge density (adjacency matrix), which are labeled graph invariants. In molecular biology, for example, a common application of the entropy is to count the number of "branchings" [9] per node by, e.g., randomly traversing a graph starting from a random point. The more extensive the branching, the greater the uncertainty of a graph's path being traversed in a unique fashion, and the higher the entropy. Thorough surveys of graph entropy are available in $[9,13,22]$, so we will avoid providing yet another one. In most, if not all, of these applications of entropy, very little attention is paid to the fact that the entropy can lead to completely disparate results depending on the ways in which the same objects of study are described, that is, due to the fact that entropy is not a graph invariant-for either labeled or unlabeled graphs_-vis-à-vis object description, a major drawback for a complexity measure [23,24] of typicality, randomness, and causality. In the survey [9], it is suggested that there is no "right" definition of entropy. Here we formally confirm this to be the case in a fundamental sense.

Indeed, the entropy requires the preselection of a graph invariant, but it is itself not a graph invariant. This is because ignorance of the probability distribution makes the entropy necessarily dependent on the graph invariant description, there being no such thing as an invariance theorem [25-27] for the Shannon entropy to provide a convergence of values independent of description language as there is in algorithmic information theory for algorithmic (Kolmogorov-Chaitin) complexity.

Definition II.9. The algorithmic complexity of an object $G$ is the length of its shortest computational description (computer program) in a reference language (of which it is independent), such that the shortest generating computer program fully reconstructs $G$ [25-28]. 

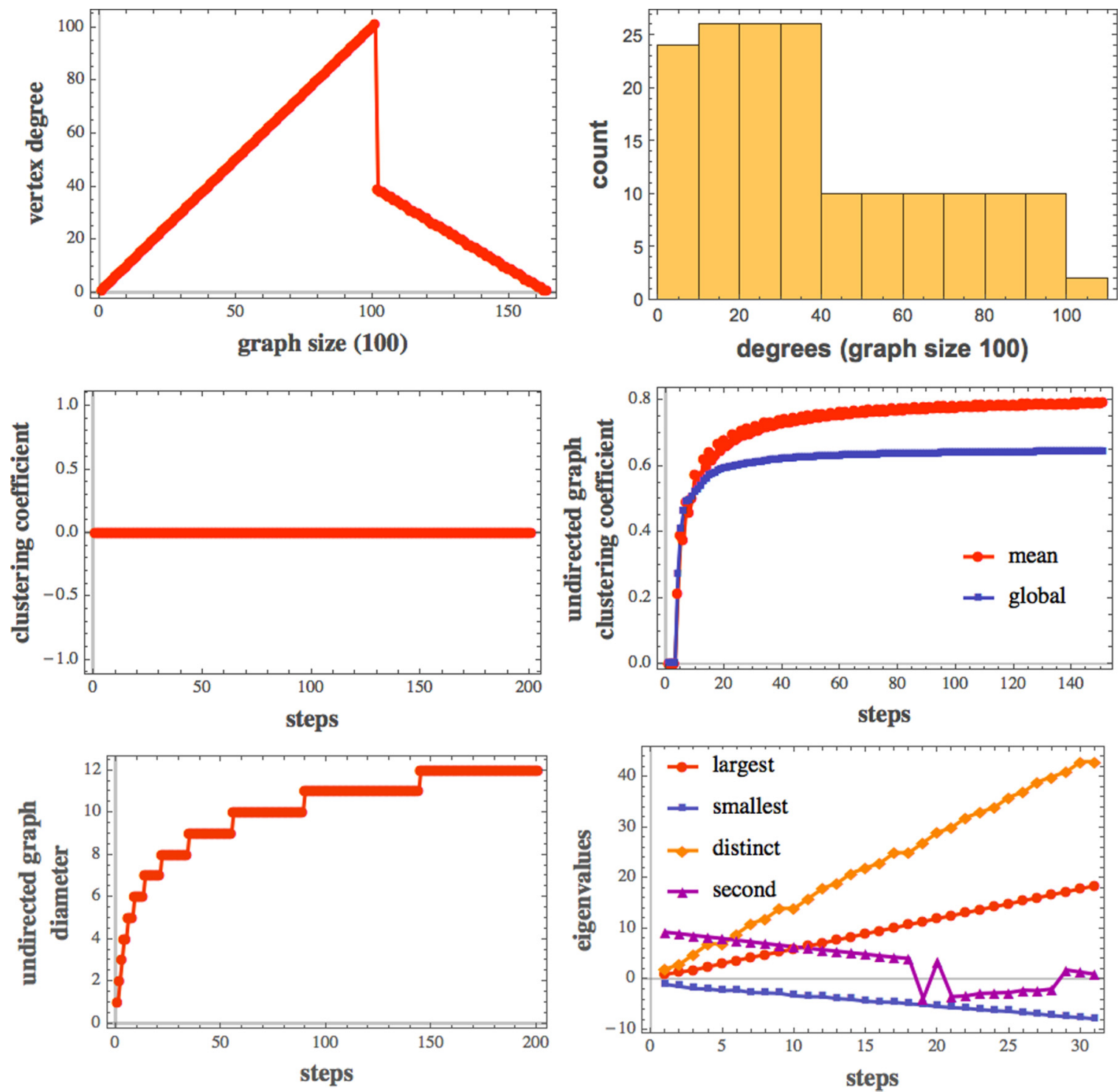

FIG. 2. Graph-theoretic and dynamic properties of the recursive $Z K$ graph. Despite the trivial construction of the recursive network, it displays all sorts of interesting convergent and divergent nontrivial graph-theoretic, dynamic, and complexity properties. For example, the clustering coefficient of the undirected graph asymptotically converges to 0.65 and some properties increase or decrease linearly while others do so polynomially. The entropy of different graph descriptions (even for fully accurate descriptions, and not because of a lack of information from the observer point of view) diverge and become trivially dependent on other simple functions (e.g., edge density or degree sequence normality). In contrast, methods based on the algorithmic probability (cf. $V$ ) assign a lower complexity to the graph than both entropy and lossless compression algorithms (e.g., Compress, depicted here) that are based on the entropy rate (word repetition). While useful for quantifying specific features of the graph that may appear interesting, no graph-theoretic or entropic measure can account for the low (algorithmic) randomness and therefore (high) causal content of the network.

\section{CONSTRUCTION OF ENTROPY-DECEIVING GRAPHS}

If we can show that we can artificially fool entropy, we will show how the entropy may fail to characterize natural or socially occurring networks: especially because, as we demonstrate, different values of the Shannon entropy can be retrieved for the same graph as functions of different features of interest of said graph, thereby showing that there is no such thing as the "Shannon entropy of a graph" but, rather, the "Shannon entropy of an identified property of a graph," which can easily be replaced with a function that simply quantifies such a property directly.

\section{A. Entropy of pseudorandom graphs}

By using integer sequences, in particular, Borel-normal irrational numbers, one can construct pseudorandom graphs, which can in turn be used to construct networks.

Definition III.1. A real number $x$ is said to be normal if all $n$-tuplets of $x$ 's digital expansion are equally likely, thereby of natural maximal $n$-order entropy rate, by the definition of Borel normality.

For example, the mathematical constant $\pi$ is believed to be an absolute Borel normal number (Borel normal in every base), and so one can take the digits of $\pi$ in any base and take 



FIG. 3. $Z K$ randomness and information content according to the lossless compression entropy and a technique, other than compression, that uses the concept of algorithmic probability to approximate the algorithmic complexity [18-20]. This means that randomness characterizations by algorithmic complexity are robust, as they are independent of object description and are, therefore, in an essential way, parameter-free, meaning that there is no need for preselection or arbitrary selection of features of interest for proper graph profiling.

$n \times n$ digits as the entries for a graph adjacency matrix of size $n \times n$ by taking $n$ consecutive segments of $n$ digits $\pi$. The resulting graph will have $n$ nodes and an edge density of 0.5 because the occurrence of 1 or 0 in $\pi$ in binary has probability 0.5 (the same as $\pi$ in decimals after transformation of digits to 0 if digit $i<5$ and 1 otherwise or $i<b / 2$ and 1 otherwise in general for any base $b$ ), thus complying with the definition of an Erdös-Rényi graph (albeit of a high density).

As theoretically predicted and numerically demonstrated in Figs. 4(a) and 4(b), the degree distribution will approximate a normal distribution around $n$. This means that the graph adjacency matrix will have maximal entropy (if $\pi$ is Borel normal) but low degree-sequence entropy because all values are around $n$ and they do not span all the possible node degrees (in particular, low degrees). This means that algorithmically constructing a graph can give rise to an object with a different entropy when the feature of interest of the said graph is changed.

A graph does not have to be of low algorithmic complexity to yield incompatible observer-dependent entropy values. One can take the digits of an $\Omega$ Chaitin number (the halting probabilities of optimal Turing machines with prefix-free domains), some of the digits of which are uncomputable. But in Fig. 4(c) we show a graph based on the first 64 digits of an $\Omega$ Chaitin number [29], thus a highest-algorithmic-complexity graph in the long run (it is ultimately uncomputable). Since randomness implies normality [30], the adjacency matrix has maximal entropy, but for the same reasons as obtained in the case of the $\pi$ graphs, it will have low degree-sequence entropy. For algorithmic complexity, in contrast, as we see in Theorem III.6, all graphs have the same algorithmic complexity regardless of their (lossless) descriptions (e.g., adjacency matrix or degree sequence), as long as the same and only the same graph (up to an isomorphism) can be reconstructed from their descriptions.

One can also start from completely different graphs. For example, Fig. 5 shows how the Shannon entropy is applied directly to the adjacency matrix as a function of the edge density, with the same entropy values retrieved despite their very different (dis)organization.

The entropy rate will be low for the regular antelope graph and higher, but still far removed from randomness, for the E-R, because by definition the degree-sequence variation of an E-R graph is small. However, in scale-free graphs the degree distribution is artificially scaled, spanning a large number of degrees as a function of the number of connected edges per added node and resulting in an overestimation of their degreesequence entropy, as can be numerically verified in Fig. 6. The degree-sequence entropy points in the opposite direction to the entropic estimation of the same graphs arrived at by looking at their adjacency matrices, when, in reality, scale-free networks produced by, e.g., Barabasi-Albert's preferential attachment algorithm [17], are recursive (algorithmic and deterministic, even if probabilities are involved), as opposed to the E-R construction built (pseudo-)randomly. The entropy of the degree sequence of scale-free graphs would suggest that they are almost as random as, or even more random than, E-R graphs for exactly the same edge densities. To circumvent this, ad hoc measures of modularity have been introduced [31], to precisely 

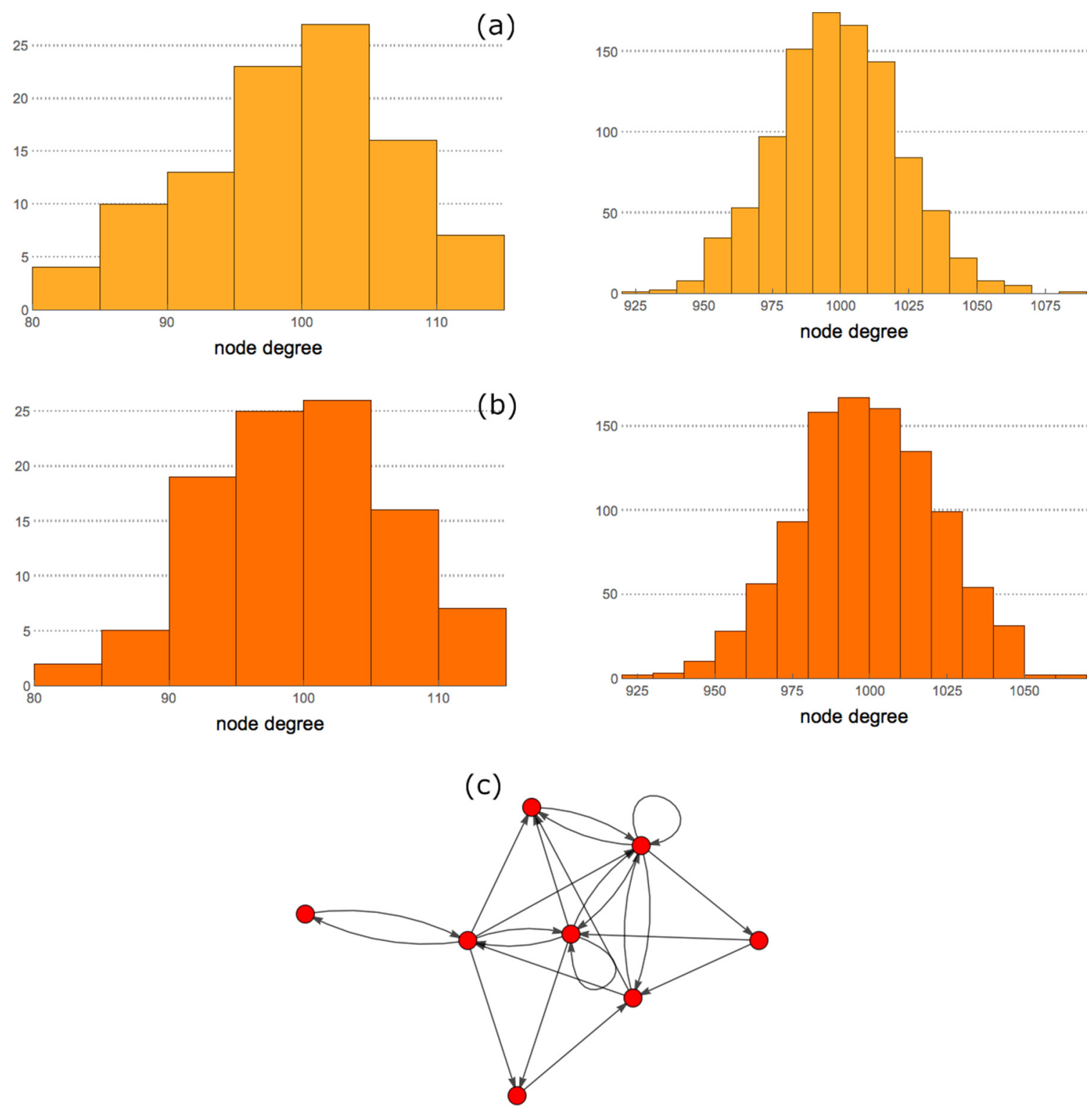

FIG. 4. Histograms of degree distributions of $\pi$ networks using 10000 (left) and $10 \times 10^{6}$ (right) digits of $\pi$ (a) in base 2 and (b) in base 10 undirected and with no self-loops. (c) A graph based on the 64 calculated bits of a partially computable $\Omega$ Chaitin number [29]. It appears to have some structure but any regularity will eventually vanish, as it is a Martin-Löf algorithmic random number [30].
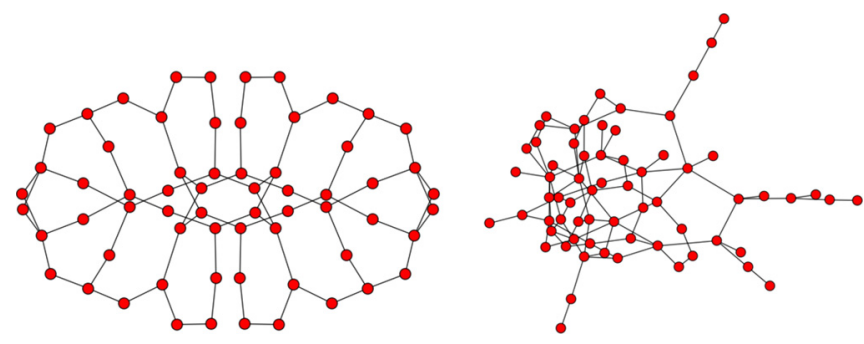

FIG. 5. A regular antelope graph (left) and an Erdös-Rényi graph (right) with the same number of edges and nodes and, therefore, the same adjacency matrix dimension and exactly the same edge density, $0.03979 \ldots$, can have very different properties. Specifically, one can be recursively (algorithmically) generated, while the other is random looking. One would wish to capture this essential difference. capture how removed a graph is from "scale-freeness" by comparing any graph to a scale-free randomized version of itself and, thereby, compelling consideration of a preselected feature of interest (scale-freeness).

Furthermore, an E-R graph can be recursively (algorithmically) generated or not, and so its Shannon entropy has no connection to the causal, algorithmic information content of the graph and can only provide clues for low-entropy graphs that can be characterized by other graph-theoretic properties, without the need for an entropic characterization.

\section{B. A low-complexity and high-entropy graph}

We introduce a method to build a family of recursive graphs with maximal entropy but low algorithmic complexity, hence graphs that appear statistically random but are, however, of 


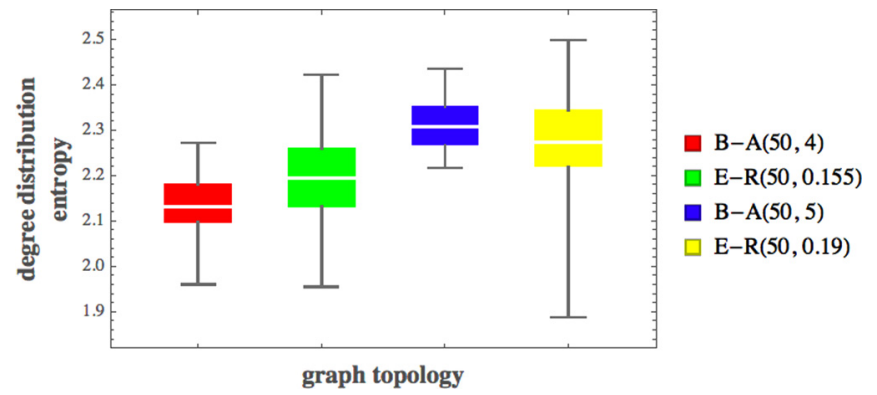

FIG. 6. Box plot of entropy values applied to the degree-sequence distribution of 10 scale-free (B-A) and 10 E-R graphs with $n=50$ nodes and the same parameters. Results may mislead as to the generative quality of each group of graphs, suggesting that B-A graphs are as random as or more random than E-R graphs, despite their recursive (causal/algorithmic and deterministic) nature, whereas in fact this should make B-A networks more random than E-R graphs. Here the E-R graphs have exactly the same edge density as the B-A graphs for four and five preferential attached edges per node. This plot illustrates how, for all purposes, the entropy can be easily fooled and cannot tell higher causal content from apparent randomness. One can always update the ensemble distribution to accommodate special cases but only after gaining knowledge by other methods.

low algorithmic randomness and thus causally (recursively) generated. Moreover, these graphs may have maximal entropy for some lossless descriptions but minimal entropy for other lossless descriptions of exactly the same objects, with both descriptions characterizing the same object and only that object, thereby demonstrating how entropy fails at unequivocally and unambiguously characterizing a graph independent of a particular feature of interest. We denote by $Z K$ a graph (unequivocally) constructed as follows:

(1) Let $1 \rightarrow 2$ be a starting graph $G$ connecting a node with label 1 to a node with label 2 . If a node with label $n$ has degree $n$, we call it a core node; otherwise, we call it a supportive node.

(2) Iteratively add a node $n+1$ to $G$ such that the number of core nodes in $G$ is maximized. The resulting graph is typified by the one in Fig. 7 .

\section{Properties of the $Z K$ graph}

The degree sequence $d$ of the labeled nodes $d=1,2, \ldots, n$ is the Champernowne constant [32] $C$ in base 10, a transcendental real whose decimal expansion is Borel normal [33], constructed by concatenating representations of successive integers whose digits are the labeled node degrees of $G$ for $n=20$ iterations (sequence A033307 in the OEIS).

The sequence of edges is a recurrence relation built upon previous iteration values between core and supportive nodes, defined by

$$
[1 / r]+[2 / r]+\cdots+[n / r],
$$

where $r=(1+\sqrt{5}) / 2$ is the golden ratio and $[\cdot]$ the floor function (sequence A183136 in the OEIS), whose values are $1,2,4,7,10,14,18,23,29,35,42,50,58,67,76,86,97,108$, $120,132,145, \ldots$.

Definition III.2. $Z K^{n}$ is a graph with at least one node with degree $x$, where $\forall x \in 1 \ldots n$.
$Z K_{m}^{n}$ is used where we want to emphasize the number of generation or time steps in the process of constructing $Z K^{n}$. The symbol $\Delta(Z K)$ denotes the maximum degree of the graph. Nodes in the $Z K$ graph belong to two types: core and supportive nodes.

Definition III.3. Node $x$ is a core node iff $\exists m \in\{1 \ldots$ $n-3\}$ such that $x \in \Delta\left(Z K_{m}^{n}\right)$. Otherwise it is a supportive node.

Theorem III.1. To convert $Z K^{r-1}$ to $Z K^{r}$, we need to add two supportive nodes to $Z K^{r-1}$ if $r$ is odd or one supportive node if $r$ is even.

Proof. By induction:

The basis: $Z K^{3}$ has three core nodes, denoted $c^{3}$, and two supportive nodes, denoted $s^{3}$. As described in the construction procedure, to convert $Z K^{3}$ to $Z K^{4}$, we choose a supportive node with maximum degree. Here, since we have only $s^{3}$ nodes, their degree is 1 . So we need to connect to three other supportive nodes. As we have only one left, we need to add two supportive nodes. Now, $Z K^{4}$ has three supportive nodes, two of them new, $s^{4}$, and one old, $s^{3}$. The old one is of degree 2 , and we need to convert it to degree 5; we have two other supportive nodes left, so we need a new supportive node, $s^{4}$. Therefore, the assumption is true for $Z K^{3}$ and $Z K^{4}$ (the basis).

Inductive step: Now, if we assume that it is true for $Z K^{n-1}$, then it is true for $Z K^{n}$.

We consider two cases:

(1) $n-1$ is odd;

(2) $n-1$ is even.

Case 1. If $n-1$ is odd, then $n-2$ is even, which means we have added one supportive node with degree 1 , and to convert $Z K^{n-1}$ to $Z K^{n}$ we need to have a core node with degree $n$. The maximum degree of a supportive node is $n-3$, and we have only one supportive node which is not connected to the core candidate node, which implies that the core candidate node will be $n-2$, and we would need to add two extra supportive nodes to our graph.

Case 2. If $n-1$ is even, then $n-2$ is odd, and therefore $Z K^{n-1}$ has two supportive nodes with degree 1 (they have only been connected to the last core nodes). So we would need to add only one node to convert the supportive node with maximum degree to a core node with degree $n$.

Corollary III.1.

(1) If $n$ is odd, then $\left|V\left(Z K^{n}\right)\right|=2 n-2$.

(2) $n-1$ is even, $\left|V\left(Z K^{n}\right)\right|=2 n-1$.

Theorem III.2. $\forall r \in 1 \ldots n$ there is a maximum of three nodes with degree $r$ in $Z K^{n}$.

Proof. By induction:

The basis: The assumption is true for $Z K^{3}$.

Inductive step: If we assume $Z K^{n-1}, \forall r \in 1 \ldots n-1$, there is a maximum of three nodes with degree $r \in Z K^{n-1}$, then $\forall r \in 1 \ldots n$; there is a maximum of three nodes with degree $r \in Z K^{n}$.

The proof is direct using Theorem.1. To generate $Z K^{n}$, we add a maximum of two supportive nodes. These nodes have degree 1 and there is no node with degree 1 except the first core node (core node with degree 1 ). Thus we have a maximum of three nodes with degree 1 . The degree of all other supportive nodes will be increased by 1 , which, based on the hypothesis of induction, has not been repeated more than three times.

Theorem III.3. ZK is of maximal degree-sequence entropy. 

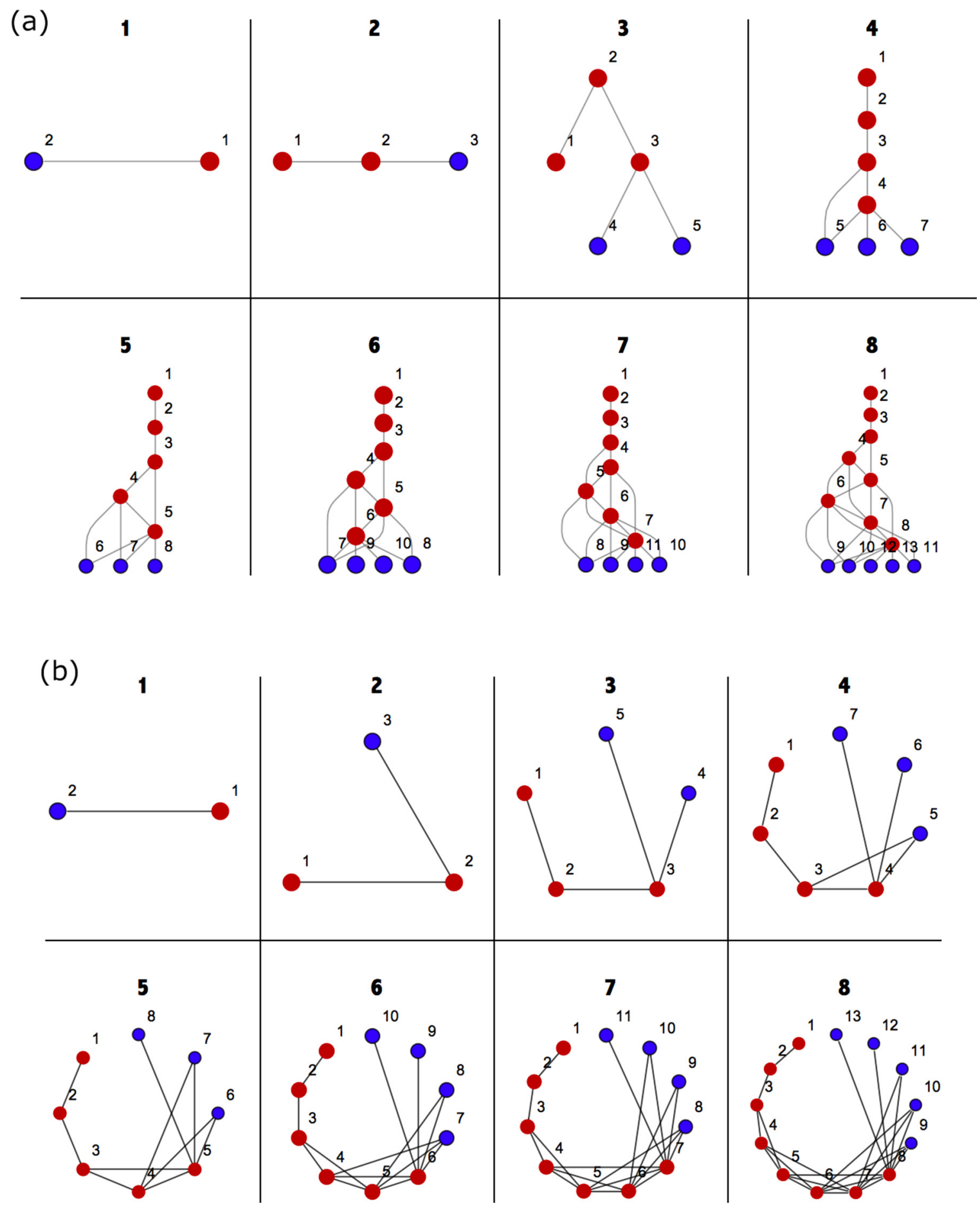

FIG. 7. (a) Treelike and (b) radial representation of the same $Z K$ graph with maximal entropy degree sequence by construction, starting from iteration 2 and proceeding to iteration 8 , adding a node at a time.

Proof. The degree sequence of the $Z K$ graph can be divided into two parts:

(1) A dominating degree subsequence associated with the core nodes (always longer than subsequence 2 of the supporting nodes) generated by the infinite series,

$$
C_{10}=\sum_{n=1}^{\infty} \sum_{k=10^{n-1}}^{10^{n}-1} \frac{k}{10^{n\left(k-10^{n-1}+1\right)+9 \sum_{\ell=1}^{n-1} 10^{\ell-1} \ell}},
$$

that produces the Champernowne constant $C_{10}$, which is Borel normal $[32,33]$.

(2) A second-degree sequence associated with the supportive nodes, whose digits do not repeat more than three times, and therefore, by Theorem III.2, it has a maximal $n$-order entropy rate for $n>2$ and a high entropy rate for $n<2$.

Therefore, the degree sequence of $Z K$ for an uninformed observer is asymptotically of maximal entropy rate for 
the natural probability distribution suggested by the degree sequence of $Z K$ itself, that is, over the set of all sequences of the same sequence length and up to the same number of alphabet elements.

Theorem III.4. The $Z K$ graph is of low algorithmic (Kolmogorov-Chaitin) complexity.

Proof. By demonstration: The computer-generated program of the $Z K$ graph written in the Wolfram language is as follows.

AddEdges [graph_] := EdgeAdd [graph, Rule@@@Distribute [\{Max [VertexDegree [graph] $]$ +1 ,

Table [i, \{i, (Max [VertexDegree [graph] ] +

2), (Max [VertexDegree [graph] ]+

1) $+(\operatorname{Max}[$ VertexDegree $[g r a p h]]+1)-$

VertexDegree [graph, Max [VertexDegree [graph] $+1]\}]\}$, List] ]

The graph can be constructed recursively for any number of nodes $n$ by nesting the AddEdges [] function as

Nest [AddEdges, Graph $[\{1->2\}, n]$

starting from the graph defined by $1 \rightarrow 2$ as the initial condition. The length of NestList with AddEdges and the initial condition in bytes is the algorithmic complexity of $Z K$, which grows by only $\log _{10} i$ and is therefore of low algorithmic randomness.

We now show that we can fully reconstruct $Z K$ from the degree sequence. As we know that we can also reconstruct $Z K$ from its adjacency matrix $[\operatorname{denoted} \operatorname{Adj}(Z K)]$, we therefore have that both are lossless descriptions from which $Z K$ can be fully reconstructed and for which the entropy provides contradictory values depending on the feature of interest.

Theorem III.5. $\forall n \in \mathbb{N}$, all instances of $Z K^{n}$ are isomorphic.

Proof. The only degree of freedom in the graph reconstruction is the selection of a supportive node to convert to a core node when there are several supportive nodes of maximal degree. As proven in Theorem III.1, the number of nodes which are added to a graph is independent of the supportive nodes selected for conversion to a core node. In any instance of a graph the numbers of nodes and edges are equal, and it is clear that by mapping the selected node in each step in any instance of a graph to the selected node in the corresponding step in another instance of $Z K$, say $Z K^{\prime}$, then $Z K^{\prime n}$, we get $f: V\left(Z K^{n}\right) \Rightarrow V\left(Z K^{\prime n}\right)$, such that $f$ is a bijection (both one-one and superimposed one on the other).

Finally, we prove that all isomorphic graphs have about the same (e.g. low) algorithmic complexity:

Theorem III.6. Let $G^{\prime}$ be an isomorphic graph of $G$. Then $K\left(G^{\prime}\right) \sim K(G)$ for all $K\left(G^{\prime}\right) \in \operatorname{Aut}(G)$, where $\operatorname{Aut}(G)$ is the automorphism group of $G$.

Proof. The idea is that if there is a significantly shorter program $p^{\prime}$ for generating $G$ compared to a program $p$ generating $\operatorname{Aut}(G)$, we can use $p^{\prime}$ to generate $\operatorname{Aut}(G)$ via $G$ and a relatively short program $c$ that tries, e.g., all permutations, and checks for isomorphism. Let us assume that there exists a program $p^{\prime}$ such that ||$p^{\prime}|-| p||>c$, i.e., the difference is not bounded by any constant, and that $K(G)=\left|p^{\prime}\right|$. We can replace $p$ with $p^{\prime}+c$ to generate $\operatorname{Aut}(G)$ such that $K(\operatorname{Aut}(G))=p^{\prime}+c$, where $c$ is a constant independent of $G^{\prime}$ that represents the size of the shortest program that generates $\operatorname{Auth}(G)$, given any $G$. Then we have that $|K(\operatorname{Aut}(G))-K(G)|<c$, which is contrary to the assumption.

The number of Borel-normal numbers that can be used as the degree sequence of a graph is determined by the necessary and sufficient conditions in [34] and [35] and is numerable infinite.

\section{Degree-sequence-targeted entropy-deceiving graph construction}

Taking advantage of the correlation between two variables $X_{1}$ and $X_{2}$ (starting independently) with the same probability distribution, let $M$ be a $2 \times 2$ matrix with the rows normalized to 1 . Consider the random variables $Y_{1}$ and $Y_{2}$, which satisfy

$$
(Y 1, Y 2)=(M . X 1, M . X 2),
$$

The correlation between $Y_{1}$ and $Y_{2}$ is just the inner product between the two rows of $M$. This can be used to generate a degree distribution of a graph with any particular entropy, provided the resulting degree sequence is graphable [34,35].

\section{GRAPH ENTROPY VERSUS GRAPH ALGORITHMIC COMPLEXITY}

The ensemble of the graphs compatible with the $Z K$ graph for the entropy of its degree distribution thus consists of the set of networks that have near-maximal degree sequence, as the sequence distribution is uninformative (nearly every degree appears only once) and thus does not reduce the statistical uncertainty, despite the algorithmic nature of the $Z K$ graph (and assuming one does not know that the graph is deterministically generated, a reasonable assumption of ignorance characteristic of the general observer in a typical, realistic case). The size of the ensemble is thereby close to $|d|$ !, the number of permutations of the elements of the degree distribution $d$ of the $Z K$ graph, constrained by the number of sequences that can actually construct a graph [34,35]. This means that, without loss of generality, any entropy-based measure (in this case applied to the degree sequence) will be misleading, assigning a high randomness after a large ensemble of equally high entropy values when it is in fact a simple recursive graph and, thereby, illustrating the limits of classical information theory for graph profiling.

\section{A. Algorithmic complexity invariance vis-à-vis full object description}

Better and complementary alternatives to graph entropy (if semicomputable) have been introduced [20,36], together with numerical methods showing that one can robustly define the algorithmic complexity (even when semicomputable) more independently of the description language not only of labeled graphs, but also of unlabeled graphs, as set forth in [18], in particular:

Definition IV.1. Algorithmic complexity of unlabeled graphs. Let $D(G)$ be a lossless description of $G$ and $\operatorname{Aut}(G)$ its automorphism group. Then

$$
K(G)=\min \{K(D(G)) \mid D(G) \in S(\operatorname{Aut}(G))\},
$$

where $K(G)$ is the algorithmic (Kolmogorov-Chaitin) complexity of the graph $G$ as introduced in Refs. [18,20] (the shortest computer program that produces $G$ upon halting) and $S(\operatorname{Aut}(G))$ is the set of all $D$ descriptions for all 
graphs in $\operatorname{Aut}(G)$, independent of $D$ (per the invariance theorem), which, unlike the graph entropy, is robust [18]. In Ref. [20], it was in fact shown that the algorithmic complexity estimation of a labeled graph is a good approximation of the algorithmic complexity of the graph automorphism group (i.e., the unlabeled graph complexity) and is correlated in one direction with the automorphism group count.

\section{B. The fragility of entropy and computable measures vis-à-vis object description}

In contrast to algorithmic complexity, no computable measure of complexity can test for all (Turing) computable regularities in a data set [30]. That is, there is no test that can be implemented as a Turing machine that takes the data as input and indicates whether they have a regularity upon halting (regularities such as "every fifth place is occupied by a consecutive prime number," to mention one example among an infinite number of possibilities).

Definition IV.2. A computable regularity is a regularity for which a test can be set as a computer program running on a specific-purpose Turing machine testing for the said regularity.

Common statistical tests, for example, are computable because they are designed to be effective, but no computable universal measure of complexity can test for every computable regularity. In other words, for every computable measure capturing a data feature $X$ intended to quantify the random content of the data, one can devise a mechanistic procedure producing $X$ that deceptively simulates the said measure for all other features.

Moreover, for every effective feature, one can devise/conceive an effective measure to test for it, but there is no computable measure able to implement a universal statistical test [30]. This means that for every effective (computable) property/feature $X$ of a computable object $S$, there is a computable measure $T$ to test for $X$ in $S$ (or any object like $S$ ), but no computable measure $T^{\prime}$ exists to test for every feature $X$ in $S$ (and all the effectively enumerable computable objects like $S$ ).

Let $D(G)$ be a lossless description of an object $G$, meaning that $G$ can be reconstructed from $D(G)$ without any loss of information. Then there is no essential distinction between $D$ and $G$ from the algorithmic point of view because $K(G)=$ $K(D(G))+c$, where $c$ is the length of the translation program (in bits) between $D$ and $G$.

Theorem IV.1. For a computable measure $H$, such as the Shannon entropy, there is no constant $c$ or logarithmic term such that $\forall G,\left|H\left(G_{D_{1}}\right)-H\left(G_{D_{2}}\right)\right|<c$ or $\mid H\left(G_{D_{1}}\right)-$ $H\left(G_{D_{2}}\right)|<\log | G \mid$ bounding the difference as a function of the size of $G$.

In other words, as we have proven by exhibiting a counterexample (the $Z K$ graph), the Shannon entropy $H$ of an object may diverge when applied to different lossless descriptions of the same object and cannot therefore be considered a robust measure of complexity. A measure of complexity should thus look not for a single property $X$ in (any possible) object $S$ but for potentially an unbounded (and potentially unidentified) number of possible properties $X=x_{0}, x_{1}, \ldots$ in any object $S$.

A sound characterization of a complexity measure can thus be established as a function that captures strictly more information about (any) $S$ than any (computable) function.
All computable functions are thus not good candidates for universal measures of complexity, as they can be replaced by a measure as a function of the property (or combination) of properties of interest and nothing else.

\section{Dependence on assumed probability distributions}

An argument against the claim that the entropy yields contradictory values when used to profile randomness (even statistical randomness) is that one can change the domain of the entropy measure in such a way as to make the entropy consistent with any possible description of a graph. For example, because we have proven that the $Z K$ algorithm is deterministic and can produce only a single $Z K$ graph, it follows that there is no uncertainty in the production of the object, there being only one graph for the formula. In this way, building a distribution of all formulas generating the $Z K$ graph will always lead to the Shannon entropy $H(Z K)=0$ for the "right" description using the "right" probability distribution containing only the $Z K$ formula(s).

According to the same argument the digits of the mathematical constant $\pi$ (to mention only the most trivial example) would have Shannon entropy $H(\pi)=0$, because the digits are produced deterministically and the right ensemble for $\pi$ should be that containing only formulas deterministically generating the digits of $\pi$, because $\pi$ is not a random variable.

Directly changing the ensemble on which the entropy operates for a specific object only facilitates conformity to some arbitrary entropy value dictated by an arbitrary expectation, e.g., that $H\left(\pi_{n}\right)=0$ for any initial segment of $\pi$ of length $n$ (entailing an entropy rate of 0 as well) because $\pi$ is deterministic and therefore no digit is surprising at all or, alternatively, $\lim _{n \rightarrow \infty} H\left(\pi_{n}\right)=\infty$ if the Shannon entropy is supposed to measure the statistical randomness of a random variable if the digits coming from $\pi$ were not known to be $\pi$. Moreover, this misbehavior has to do not with a lack of knowledge but with the lack of an invariance theorem, because $\pi$ is deterministically generated and hence its digits do not fundamentally reduce the uncertainty. But if one assumes that the digits of $\pi$ do not look stochastic in order to assign it a Shannon entropy equal to 0 , then one is forced to concede that even perfect statistical randomness, produced by a supposedly Borel-normal number, has, in objective terms, a Shannon entropy (and entropy rate) equal to 0 , but the highest Shannon entropy (and entropy rate) from an observer perspective (as it will never be certain that the streaming digits are truly $\pi$ ). In other words, the asymptotic behavior of a random variable producing the digits of $\pi$ in base $n$ will approximate maximum Shannon entropy over the probability space of all possible $n$-ary sequences, but $\pi$ itself has Shannon entropy 0 because it is not a random variable.

\section{An algorithmic maximum entropy model}

Following the statistical mechanics approach [4], a typical recursively generated graph such as the $Z K$ graph would, based on its degree sequence, be characterized as being typically random from the observer perspective-because the Shannon entropy will find the graph to be statistically random and thus just as random as any member of the set of all graphs with a (near-) maximal degree-sequence entropy-thus giving no 
indication of the actual recursive nature of the $Z K$ graph and misleading the observer.

In contrast, the type of approach introduced in [20], based upon trying to find clues to the recursive nature of an object such as a graph, would asymptotically find the causal nature of a recursively generating object such as the $Z K$ graph, independent of probability distributions, even if it is more difficult to estimate.

Rectifying the approaches based on models of maximum entropy involves updating and replacing the assumption of the maximum entropy ensemble. An example illustrating how to achieve this in the context of, e.g., a Bayesian approach, has been provided in [37] and consists in replacing the uninformative prior with the uninformative algorithmic probability distribution, the so-called universal distribution, as introduced by Levin [28]. The general approach has already delivered some important results [38] by, e.g., quantifying the degree of human cognitive randomness that previous statistical approaches and measures such as the entropy made it impossible to quantify. Animated videos have been made available explaining applications to graph complexity (https://youtu.be/E238zKsPCgk) and to cognition in the context of random generation tasks (https://youtu.be/E-YjBE5qm7c). A tool has also been placed online (http://complexitycalculator.com/) for sequences and arrays, and thus the reader can experiment with an actual numerical tool and explore the differences between the statistical and the algorithmic approaches.

\section{CONCLUSIONS}

The methods introduced here allow the construction of "Borel-normal pseudorandom graphs," uncomputable number-based graphs, and algorithmically produced graphs, while illustrating the shortcomings of computable graph-theoretic and entropy approaches to graph complexity beyond random feature selection and their failure when it comes to profiling randomness and hence causal content (as opposed to randomness).

We have shown that the entropy is highly observer dependent even in the face of full accuracy and access to lossless object descriptions and thus has to be complemented with measures of algorithmic content. We have produced specific complexity-deceiving graphs for which the entropy retrieves disparate values when an object is described differently (thus with different underlying distributions), even when the descriptions reconstruct exactly the same, and only the same, object. This drawback of the Shannon entropy, ultimately related to its dependence on the distribution, is all the more serious because it is easily overlooked in the case of objects other than strings, for instance, graphs. For an object such as a graph, we have shown that changing the descriptions may not only change the values but actually produce divergent, contradictory values.
We constructed a $Z K$ graph about which the following is true when it is described by its adjacency matrix $\operatorname{Adj}(Z K)$ : $\lim _{n \rightarrow \infty} H\left(\operatorname{Adj}\left(Z K_{n}\right)\right)=0$ for increasing graph size $n$. Contradictorily, considering the same $Z K$ graph degree sequence, we found that $\lim _{n \rightarrow \infty} H\left(\operatorname{Seq}\left(Z K_{n}\right)\right)=\infty$ for the same growth rate $n$, even though both $\operatorname{Adj}\left(G_{n}\right)$ and $\operatorname{Seq}\left(G_{n}\right)$ are lossless descriptions of the same graph that construct exactly the same $Z K$ graph, and only a $Z K$ graph.

This means not only that one needs to choose a description of interest in order to apply a definition of entropy, such as the adjacency matrix of a network (or its incidence or Laplacian matrix) or its degree sequence, but that as soon as the choice is made, the entropy becomes a trivial counting function of the specific feature of interest, and of that feature alone. In the case of, for example, the adjacency matrix of a network (or any related matrix associated with the graph, such as the incidence or Laplacian matrix), the entropy becomes a function of the edge density, while for the degree sequence, the entropy becomes a function of the sequence normality. The entropy can thus trivially be replaced by such functions without any loss, but it cannot be used to profile the object (randomness, or information content) in any way independent of an arbitrary feature of interest.

These results and observations have far-reaching consequences. For example, recent literature appears contradictory, by turns suggesting that cancer cells display an increase in entropy [39] and reporting that cancer cells display a decrease in entropy [40], in both cases applied to a function of the degree distribution over networks of molecular interactions. Cells are also believed to be in a state of criticality between evolvability and robustness [41,42], which may make them look random though they are not. This means that the entropy may be overestimating the randomness in the best case or misleading in the worst case, as we have found in the instance of disparate values for the same objects, thus suggesting that additional safeguards are needed to achieve consistency and soundness.

New developments $[18,20]$ promise more robust complementary measures of (graph) complexity less dependent on object description, measures based upon the mathematical theory of randomness and algorithmic probability, which are better equipped to profile the causality and algorithmic information content and cover the statistical randomness and thus can be considered an observer-improved generalization of the Shannon entropy.

\section{ACKNOWLEDGMENTS}

N.A.K. was supported by a Vinnova VINNMER fellowship, Stratneuro. H.Z. was supported by the Swedish Research Council (VR).

H.Z. and N.A.K. contributed equally to this paper.
[1] S. Boccaletti et al., The structure and dynamics of multilayer networks, Phys. Rep. 544, 1 (2014).

[2] Z. Chen, M. Dehmer, F. Emmert-Streib, and Y. Shi, Entropy bounds for dendrimers, Appl. Math. Comput. 242, 462 (2014).
[3] C. Orsini et al., Quantifying randomness in real networks, Nat. Commun. 6, 8627 (2015).

[4] G. Bianconi, The entropy of randomized network ensembles, Europhys. Lett. 81, 28005 (2008). 
[5] J. Korner and K. Marton, Random access communication and graph entropy, IEEE Trans. Info. Theory 34, 312 (1988).

[6] M. Dehmer, S. Borgert, and F. Emmert-Streib, Entropy bounds for hierarchical molecular networks, PLoS One 3, e3079 (2008).

[7] G. Lu, B. Li, and L. Wang, Some new properties for degree-based graph entropies, Entropy 17, 8217 (2015).

[8] A. Mowshowitz, Entropy and the complexity of graphs: I. An index of the relative complexity of a graph, Bull. Math. Biophys. 30, 175 (1968).

[9] A. Mowshowitz and M. Dehmer, Entropy and the complexity of graphs revisited, Entropy 14, 559 (2012).

[10] J. Ziv and A. Lempel, Compression of individual sequences via variable-rate coding, IEEE Trans. Info. Theory 24, 530 (1978).

[11] T. Böttcher, J. Chem. Inf. Model. 56, 462 (2016).

[12] E. Estrada, A. José, and N. Hatano, Walk entropies in graphs, Lin. Algebra Appl. 443, 235 (2014).

[13] M. Dehmer and A. Mowshowitz, A history of graph entropy measures, Info. Sci. 181, 57 (2011).

[14] D. C. Sengupta and J. D. Sengupta, Application of graph entropy in CRISPR and repeats detection in DNA sequences, Comput. Mol. Biosci. 6, 41 (2016).

[15] E. Estrada, Quantifying network heterogeneity, Phys. Rev. E 82, 066102 (2010).

[16] P. Erdos and A. Rényi, On the evolution of random graphs, Publ. Math. Inst. Hung. Acad. Sci. 5, 17 (1960).

[17] A.-L. Barabási and R. Albert, Emergence of scaling in random networks, Science 286, 509 (1999).

[18] H. Zenil, N. A. Kiani, and J. Tegnér, Methods of information theory and algorithmic complexity for network biology, Semin. Cell Dev. Biol. 51, 32 (2016).

[19] H. Zenil, F. Soler-Toscano, J.-P. Delahaye, and N. Gauvrit, Two-dimensional Kolmogorov complexity and an empirical validation of the coding theorem method by compressibility, Peer J. Comput. Sci. 1, e23 (2015).

[20] H. Zenil, F. Soler-Toscano, K. Dingle, and A. A. Louis, Correlation of automorphism group size and topological properties with program-size complexity evaluations of graphs and complex networks, Physica A: Stat. Mech. Appl. 404, 341 (2014).

[21] C. E. Shannon, A mathematical theory of communication, Bell Syst. Tech. J. 27, 379,623 (1948) [Math. Rev. (MathSciNet) MR10, 133e (1948)].

[22] G. Simonyi, Graph entropy: A survey, Combin. Opt. 20, 399 (1995).

[23] H. Zenil, Small data matters, correlation versus causation and algorithmic data analytics, in Predictability in the World: Philosophy and Science in the Complex World of Big Data (Springer-Verlag, Berlin, 2013).

[24] H.r Zenil, F. Soler-Toscano, N. A. Kiani, S. Hernández-Orozco, and A. Rueda-Toicen, A decomposition method for global evaluation of Shannon entropy and local estimations of algorithmic complexity, submitted for publication (2016).

[25] G. J. Chaitin, On the length of programs for computing finite binary sequences, J. ACM (JACM) 13, 547 (1966).

[26] A. N. Kolmogorov, Three approaches to the quantitative definition of information, Int. J. Comput. Math. 2, 157 (1968).

[27] R. J. Solomonoff, A formal theory of inductive inference. Part i, Info. Control 7, 1 (1964).

[28] L. A. Levin, Laws of information conservation (nongrowth) and aspects of the foundation of probability theory, Prob. Peredachi Inf. 10, 30 (1974).

[29] C. S. Calude, M. J. Dinneen, C.-K. Shu et al., Computing a glimpse of randomness, Exp. Math. 11, 361 (2002).

[30] P. Martin-Löf, The definition of random sequences, Info. Control 9, 602 (1966).

[31] R. V. Solé and S. Valverde, Spontaneous emergence of modularity in cellular networks, J. Roy. Soc. Interf. 5, 129 (2008).

[32] D. G. Champernowne, The construction of decimals normal in the scale of ten, J. London Math. Soc. 1, 254 (1933).

[33] E. Borel, Les probabilités dénombrables et leurs applications arithmétiques, Rend. Circ. Mat. Palermo (1884-1940) 27, 247 (1909).

[34] H. Kim, C. I. Del Genio, K. E. Bassler, and Z. Toroczkai, Degreebased graph construction, New J. Phys. 14, 023012 (2012).

[35] H. Kim, Z. Toroczkai, P. L. Erdôs, I. Miklós, and L. A. Székely, Degree-based graph construction, J. Phys. A: Math. Theor. 42, 392001 (2009).

[36] F. Soler-Toscano, H. Zenil, J.-P. Delahaye, and N. Gauvrit, Calculating Kolmogorov complexity from the output frequency distributions of small Turing machines, PloS One 9, e96223 (2014).

[37] Representation and Reality in Humans, Other Living Organisms and Intelligent Machines, Studies in Applied Philosophy, Epistemology and Rational Ethics, edited by G. Dodig-Crnkovic and R. Giovagnoli, Vol. 28 (Springer-Verlag, 2017), p. XIII, 315.

[38] N. Gauvrit, H. Zenil, F. Soler-Toscano, J.-P. Delahaye, and P. Bruger, Human behavioral complexity peaks at age 25, PLoS Comput. Biol. 13, e1005408 (2017).

[39] A. E. Teschendorff and S. Severini, Increased entropy of signal transduction in the cancer metastasis phenotype, BMC Syst. Biol. 4, 104 (2010).

[40] J. West, G. Bianconi, S. Severini, and A. E. Teschendorff, Differential network entropy reveals cancer system hallmarks, Sci. Rep. 2, 802 (2012).

[41] P. Csermely et al., Cancer stem cells display extremely large evolvability: Alternating plastic and rigid networks as a potential mechanism, Semin. Cancer Biol. 30, 42 (2015).

[42] C. Torres-Sosa, S. Huang, and M. Aldana, Criticality is an emergent property of genetic networks that exhibit evolvability, PLoS Comput. Biol. 8, e1002669 (2012). 